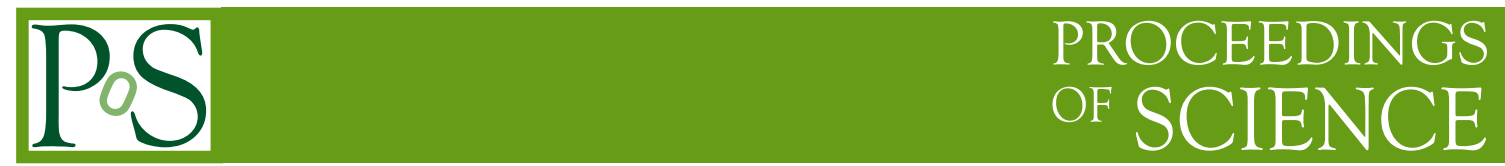

\title{
Perturbative Finiteness of Superstring Theory
}

\author{
Nathan Berkovits* \\ IFT/UNESP, São Paulo, Brazil \\ E-mail: nberkovi@ift.unesp.br
}

In these proceedings, the multiloop amplitude prescription using the super-Poincare invariant pure spinor formalism for the superstring is reviewed. Unlike the RNS prescription, there is no sum over spin structures and surface terms coming from the boundary of moduli space can be ignored. Massless $N$-point multiloop amplitudes vanish for $N<4$, which implies (with two mild assumptions) the perturbative finiteness of superstring theory. And $R^{4}$ terms receive no multiloop contributions in agreement with the Type IIB $S$-duality conjecture of Green and Gutperle.

Fourth International Winter Conference on Mathematical Methods in Physics 09 - 13 August 2004

Centro Brasileiro de Pesquisas Fisicas (CBPF/MCT), Rio de Janeiro, Brazil

${ }^{*}$ Speaker. 


\section{Introduction}

The computation of multiloop amplitudes in superstring theory has many important applications such as verifying perturbative finiteness and testing duality conjectures. Nevertheless, this subject has received little attention over the last fifteen years, mainly because of difficulties in computing multiloop amplitudes using either the Ramond-Neveu-Schwarz (RNS) or Green-Schwarz (GS) formalism.

In the RNS formalism, spacetime supersymmetric amplitudes are obtained after summing over spin structures, which can be done explicitly only when the number of loops and external states is small [1]. Since there are divergences near the boundary of moduli space before summing over spin structures, surface terms in the amplitude expressions need to be treated with care [2][3][4] [5]. Furthermore, the complicated nature of the Ramond vertex operator in the RNS formalism [6] makes it difficult to compute amplitudes involving external fermions or Ramond-Ramond bosons. For these reasons, up to now, explicit multiloop computations in the RNS formalism have been limited to four-point two-loop amplitudes involving external Neveu-Schwarz bosons [7] [5].

In the GS formalism, spacetime supersymmetry is manifest but one needs to fix light-cone gauge and introduce non-covariant operators at the interaction points of the Mandelstam string diagram [8] [9] [10]. Because of complications caused by these non-covariant interaction point operators [11], explicit amplitude expressions have been computed using the light-cone GS formalism only for four-point tree and one-loop amplitudes [8].

Over the past twenty years, there have been several approaches to covariant quantization of the superstring. However, none of these approaches were able to compute even tree-level amplitudes in a super-Poincaré covariant manner. Four years ago, a new formalism for the superstring was proposed [12] [13] with manifest ten-dimensional super-Poincaré covariance. In conformal gauge, the worldsheet action is quadratic and physical states are defined using a BRST operator constructed from superspace matter variables and a pure spinor ghost variable. A super-Poincaré covariant prescription was given for computing $N$-point tree amplitudes, which was shown to coincide with the standard RNS prescription [14] [15]. It was also proven that the BRST cohomology reproduces the correct superstring spectrum [16] and that BRST invariance in a curved supergravity background implies the low-energy superspace equations of motion for the background superfields [17] [18].

Because of the pure spinor constraint satisfied by the worldsheet ghosts, it was not obvious how to define functional integration in this formalism. For this reason, the tree amplitude prescription in [12] relied on BRST cohomology for defining the correct normalization of the worldsheet zero modes. Furthermore, there was no natural $b$ ghost in this formalism, which made it difficult to define amplitudes in a worldsheet reparameterization-invariant manner. Because of these complications, it was not clear how to compute loop amplitudes using this formalism and other groups looked for ways of relaxing the pure spinor constraint without modifying the BRST cohomology [19][20][21].

Recently, it was shown how to perform functional integration by defining a Lorentz-invariant measure for the pure spinor ghosts, introducing appropriate "picture-changing" operators, and constructing a composite $b$ ghost in a non-zero picture. With these three ingredients, it was straightforward to generalize the tree amplitude prescription of [12] to a super-Poincaré covariant prescription for $N$-point $g$-loop amplitudes [22]. 
The need for picture-changing operators in this formalism is not surprising since, like the bosonic $(\beta, \gamma)$ ghosts in the RNS formalism [6], the pure spinor ghosts are chiral bosons with worldsheet zero modes. As in the RNS formalism, the worldsheet derivatives of these picture-changing operators are BRST trivial so, up to possible surface terms, the amplitudes are independent of their locations on the worldsheet. But unlike the RNS formalism, there is no need to sum over spin structures and there are no divergences at the boundary of moduli space. So surface terms can be safely ignored in the loop amplitude computations.

Although the explicit computation of arbitrary loop amplitudes is complicated, there are several features of the prescription which are simpler than in the RNS prescription. For example, there is no sum over spin structures, no surface terms from the boundary of moduli space, and no unphysical poles from negative-energy chiral bosons. Furthermore, the partition functions for the matter and ghost variables cancel, amplitudes involving external Ramond states are no more complicated than those involving external Neveu-Schwarz states, and one can easily prove vanishing theorems by counting zero modes of the fermionic superspace variables. For example, S-duality of the Type IIB superstring implies that $R^{4}$ terms in the low-energy effective action receive no perturbative corections above one-loop [23]. After much effort, this was recently verified in the RNS formalism at two-loops [7][5]. Using the formalism described here, this S-duality conjecture can be easily verified for all loops.

Similarly, one can easily prove the non-renormalization theorem that massless $\mathrm{N}$-point multiloop amplitudes vanish whenever $N<4$. Assuming factorization, this non-renormalization theorem implies the absence of divergences near the boundary of moduli space [4] [24]. Note that the boundary of moduli space includes two types of degenerate surfaces: surfaces where the radius $R$ of a handle shrinks to zero, and surfaces which split into two worldsheets connected by a thin tube. As explained in [4], the first type of degenerate surface does not lead to divergent amplitudes in a tachyon-free theory since, after including the $\log (R)$ dependence coming from integration over the loop momenta, the amplitude integrand diverges slower than $1 / R$. The second type of degenerate surface can lead to a divergent amplitude if there is an onshell state propagating along the thin tube between the two worldsheets. But when all external states are on one of the two worldsheets, vanishing of the one-point function implies the absence of this divergence. And when all but one of the external states are on one of the two worldsheets, vanishing of the two-point function implies the absence of this divergence. Finally, when there are at least two external states on each of the two worldsheets, the divergence can be removed by analytic continuation of the external momenta [4]. Note that vanishing of the three-point function is not required for finiteness.

So with the two mild assumptions of factorization and absence of unphysical divergences in the interior of moduli space ${ }^{1}$, this non-renormalization theorem implies that massless multiloop superstring amplitudes are finite order-by-order in perturbation theory. Previous attempts to prove this non-renormalization theorem using the RNS formalism [24] were unsuccessful because they ignored unphysical poles of the spacetime supersymmetry currents [2] and incorrectly assumed that the integrand of the scattering amplitude was spacetime supersymmetric. Using the GS formalism,

\footnotetext{
${ }^{1}$ In light-cone gauge, unphysical divergences in the interior of moduli space could come from singularities between colliding interaction points [11] [25]. In conformal gauge, there are no obvious potential sources for these unphysical divergences in the interior of moduli space since the amplitudes are independent (up to surface terms) of the locations of picture-changing operators.
} 
there are arguments for the non-renormalization theorem [26], however, these arguments do not rule out the possibility of unphysical divergences in the interior of moduli space from contact term singularities between light-cone interaction point operators [11]. Mandelstam was able to overcome this obstacle and prove finiteness [25] by combining different features of the RNS and GS formalisms. However, the finiteness proof here is more direct than the proof of [25] since it is derived from a single formalism.

In section 2 of this paper, the worldsheet action and BRST operator in the super-Poincaré invariant pure spinor formalism of [12] are reviewed. In section 3, the three new ingredients needed for multiloop amplitude computations are described: Lorentz-invariant measure factors for the pure spinor ghosts; picture-changing operators; and a composite $b$ ghost in non-zero picture. In section 4, a super-Poincaré covariant prescription is given for $N$-point $g$-loop amplitudes which has been shown to agree with the RNS prescription for tree and massless four-point one-loop amplitudes. (See [22] for a more detailed version of sections 3 and 4.) In section 5, the counting of fermionic zero modes is used to prove certain vanishing theorems. And in section 6, some open questions and further applications are discussed.

\section{Review of Super-Poincaré Invariant Pure Spinor Formalism}

\subsection{Worldsheet action}

The worldsheet variables in the Type IIB version of this formalism include the Green-SchwarzSiegel [27][28] matter variables $\left(x^{m}, \theta^{\alpha}, p_{\alpha} ; \bar{\theta}^{\alpha}, \bar{p}_{\alpha}\right)$ for $m=0$ to 9 and $\alpha=1$ to 16 , and the pure spinor ghost variables $\left(\lambda^{\alpha}, w_{\alpha} ; \bar{\lambda}^{\alpha}, \bar{w}_{\alpha}\right)$ where $\lambda^{\alpha}$ and $\bar{\lambda}^{\alpha}$ are constrained to satisfy the pure spinor conditions

$$
\lambda^{\alpha}\left(\gamma^{m}\right)_{\alpha \beta} \lambda^{\beta}=0, \quad \bar{\lambda}^{\alpha}\left(\gamma^{m}\right)_{\alpha \beta} \bar{\lambda}^{\beta}=0
$$

for $m=0$ to 9 . $\left(\gamma^{m}\right)_{\alpha \beta}$ and $\left(\gamma^{m}\right)^{\alpha \beta}$ are $16 \times 16$ symmetric matrices which are the off-diagonal blocks of the $32 \times 32$ ten-dimensional $\Gamma$-matrices and satisfy $\left(\gamma^{(m}\right)_{\alpha \beta}\left(\gamma^{n)}\right)^{\beta \gamma}=2 \eta^{m n} \delta_{\alpha}^{\gamma}$. For the Type IIA version of the formalism, the chirality of the spinor indices on the right-moving variables is reversed, and for the heterotic version, the right-moving variables are the same as in the RNS formalism.

In conformal gauge, the worldsheet action is

$$
S=\int d^{2} z\left[-\frac{1}{2} \partial x^{m} \bar{\partial} x_{m}-p_{\alpha} \bar{\partial} \theta^{\alpha}-\bar{p}_{\alpha} \partial \bar{\theta}^{\alpha}+w_{\alpha} \bar{\partial} \lambda^{\alpha}+\bar{w}_{\alpha} \partial \bar{\lambda}^{\alpha}\right]
$$

where $\lambda^{\alpha}$ and $\bar{\lambda}^{\alpha}$ satisfy (2.1). The OPE's for the matter variables are easily computed to be

$$
x^{m}(y) x^{n}(z) \rightarrow-\eta^{m n} \log |y-z|^{2}, \quad p_{\alpha}(y) \theta^{\beta}(z) \rightarrow(y-z)^{-1} \delta_{\alpha}^{\beta}
$$

however, the pure spinor constraint on $\lambda^{\alpha}$ prevents a direct computation of its OPE's with $w_{\alpha}$. As discussed in [12], one can solve the pure spinor constraint and express $\lambda^{\alpha}$ in terms of eleven unconstrained free fields which manifestly preserve a U(5) subgroup of the (Wick-rotated) Lorentz group. Although the OPE's of the unconstrained variables are not manifestly Lorentz-covariant, all OPE computations involving $\lambda^{\alpha}$ can be expressed in a manifestly Lorentz-covariant manner. 
So the non-covariant unconstrained description of pure spinors is useful only for verifying certain coefficients in the Lorentz-covariant OPE's.

Because of the pure spinor constraint on $\lambda^{\alpha}$, the worldsheet variables $w_{\alpha}$ contain the gauge invariance

$$
\delta w_{\alpha}=\Lambda^{m}\left(\gamma_{m} \lambda\right)_{\alpha},
$$

so 5 of the 16 components of $w_{\alpha}$ can be gauged away. To preserve this gauge invariance, $w_{\alpha}$ can only appear in the gauge-invariant combinations

$$
N_{m n}=\frac{1}{2} w_{\alpha}\left(\gamma_{m n}\right)^{\alpha} \lambda^{\beta}, \quad J=w_{\alpha} \lambda^{\alpha},
$$

which are the Lorentz currents and ghost current. As shown in [15] and [16] using either the U(5) or $\mathrm{SO}(8)$ unconstrained descriptions of pure spinors, $N_{m n}$ and $J$ satisfy the Lorentz-covariant OPE's

$$
\begin{gathered}
N_{m n}(y) \lambda^{\alpha}(z) \rightarrow \frac{1}{2}(y-z)^{-1}\left(\gamma_{m n} \lambda\right)^{\alpha}, \quad J(y) \lambda^{\alpha}(z) \rightarrow(y-z)^{-1} \lambda^{\alpha}, \\
N^{k l}(y) N^{m n}(z) \rightarrow-3(y-z)^{-2}\left(\eta^{n[k} \eta^{l] m}\right)+(y-z)^{-1}\left(\eta^{m[l} N^{k] n}-\eta^{n[l} N^{k] m}\right), \\
J(y) J(z) \rightarrow-4(y-z)^{-2}, \quad J(y) N^{m n}(z) \rightarrow \text { regular, } \\
N_{m n}(y) T(z) \rightarrow(y-z)^{-2} N_{m n}(z), \quad J(y) T(z) \rightarrow-8(y-z)^{-3}+(y-z)^{-2} J(z),
\end{gathered}
$$

where

$$
T=-\frac{1}{2} \partial x^{m} \partial x_{m}-p_{\alpha} \partial \theta^{\alpha}+w_{\alpha} \partial \lambda^{\alpha}
$$

is the left-moving stress tensor. From the OPE's of (2.6), one sees that the pure spinor condition implies that the levels for the Lorentz and ghost currents are -3 and -4 , and that the ghost-number anomaly is -8 . Note that the total Lorentz current $M^{m n}=-\frac{1}{2}\left(p \gamma^{m n} \theta\right)+N^{m n}$ has level $k=4-3=$ 1 , which coincides with the level of the RNS Lorentz current $M^{m n}=\psi^{m} \psi^{n}$. The ghost-number anomaly of -8 will be related in subsection (3.1) to the pure spinor measure factor. Finally, the stress tensor of (2.7) has no central charge since the $(+10-32)$ contribution from the $\left(x^{m}, \theta^{\alpha}, p_{\alpha}\right)$ variables is cancelled by the +22 contribution from the eleven independent $\left(\lambda^{\alpha}, w_{\alpha}\right)$ variables.

\subsection{BRST operator and massless vertex operators}

Physical open string states in this formalism are defined as super-Poincaré covariant states of ghost-number +1 in the cohomology of the nilpotent BRST-like operator

$$
Q=\oint \lambda^{\alpha} d_{\alpha}
$$

where

$$
d_{\alpha}=p_{\alpha}-\frac{1}{2} \gamma_{\alpha \beta}^{m} \theta^{\beta} \partial x_{m}-\frac{1}{8} \gamma_{\alpha \beta}^{m} \gamma_{m} \gamma_{\delta} \theta^{\beta} \theta^{\gamma} \partial \theta^{\delta}
$$

is the supersymmetric Green-Schwarz constraint. As shown by Siegel [28], $d_{\alpha}$ satisfies the OPE's

$$
\begin{gathered}
d_{\alpha}(y) d_{\beta}(z) \rightarrow-(y-z)^{-1} \gamma_{\alpha \beta}^{m} \Pi_{m}, \quad d_{\alpha}(y) \Pi^{m}(z) \rightarrow(y-z)^{-1} \gamma_{\alpha \beta}^{m} \partial \theta^{\beta}(z), \\
d_{\alpha}(y) \partial \theta^{\beta}(z) \rightarrow(y-z)^{-2} \delta_{\alpha}^{\beta}, \quad \Pi^{m}(y) \Pi^{n}(z) \rightarrow-(y-z)^{-2} \eta^{m n},
\end{gathered}
$$


where $\Pi^{m}=\partial x^{m}+\frac{1}{2} \theta \gamma^{m} \partial \theta$ is the supersymmetric momentum and

$$
q_{\alpha}=\oint\left(p_{\alpha}+\frac{1}{2} \gamma_{\alpha \beta}^{m} \theta^{\beta} \partial x_{m}+\frac{1}{24} \gamma_{\alpha \beta}^{m} \gamma_{m} \gamma \delta \theta^{\beta} \theta^{\gamma} \partial \theta^{\delta}\right)
$$

is the supersymmetric generator satisfying

$$
\left\{q_{\alpha}, q_{\beta}\right\}=\gamma_{\alpha \beta}^{m} \oint \partial x_{m}, \quad\left[q_{\alpha}, \Pi^{m}(z)\right]=0, \quad\left\{q_{\alpha}, d_{\beta}(z)\right\}=0 .
$$

To compute the massless spectrum of the open superstring, note that the most general vertex operator with zero conformal weight at zero momentum and +1 ghost-number is

$$
V=\lambda^{\alpha} A_{\alpha}(x, \theta)
$$

where $A_{\alpha}(x, \theta)$ is a spinor superfield depending only on the worldsheet zero modes of $x^{m}$ and $\theta^{\alpha}$. Using the OPE that $d_{\alpha}(y) f(x(z), \theta(z)) \rightarrow(y-z)^{-1} D_{\alpha} f$ where

$$
D_{\alpha}=\frac{\partial}{\partial \theta^{\alpha}}+\frac{1}{2} \theta^{\beta} \gamma_{\alpha \beta}^{m} \partial_{m}
$$

is the supersymmetric derivative, one can easily check that $Q V=0$ and $\delta V=Q \Lambda$ implies that $A_{\alpha}(x, \theta)$ must satisfy $\lambda^{\alpha} \lambda^{\beta} D_{\alpha} A_{\beta}=0$ with the gauge invariance $\delta A_{\alpha}=D_{\alpha} \Lambda$. But $\lambda^{\alpha} \lambda^{\beta} D_{\alpha} A_{\beta}=0$ implies that

$$
D_{\alpha} A_{\beta}+D_{\beta} A_{\alpha}=\gamma_{\alpha \beta}^{m} A_{m}
$$

for some vector superfield $A_{m}$ with the gauge transformations

$$
\delta A_{\alpha}=D_{\alpha} \Lambda, \quad \delta A_{m}=\partial_{m} \Lambda .
$$

In components, one can use (2.13) and (2.14) to gauge $A_{\alpha}$ and $A_{m}$ to the form

$$
\begin{gathered}
A_{\alpha}(x, \theta)=e^{i k \cdot x}\left(\frac{1}{2} a_{m}\left(\gamma^{m} \theta\right)_{\alpha}-\frac{1}{3}\left(\xi \gamma_{m} \theta\right)\left(\gamma^{m} \theta\right)_{\alpha}+\ldots\right), \\
A_{m}(x, \theta)=e^{i k \cdot x}\left(a_{m}+\left(\xi \gamma^{m} \theta\right)+\ldots\right),
\end{gathered}
$$

where $k^{2}=k^{m} a_{m}=k^{m}\left(\gamma_{m} \xi\right)_{\alpha}=0$, and ... involves products of $k_{m}$ with $a_{m}$ or $\xi^{\alpha}$. So (2.13) and (2.14) are the equations of motion and gauge invariances of the ten-dimensional super-Maxwell multiplet, and the cohomology at ghost-number +1 of $Q$ correctly describes the massless spectrum of the open superstring [29].

As in bosonic string theory, one can obtain the integrated open string vertex operator $\int d z U(z)$ from the unintegrated vertex operator $V$ by requiring that $Q U(z)=\partial V(z)$. For the massless states where the unintegrated vertex operator is $V=\lambda^{\alpha} A_{\alpha}(x, \theta)$, one finds that

$$
U=\partial \theta^{\alpha} A_{\alpha}(x, \theta)+\Pi^{m} A_{m}(x, \theta)+d_{\alpha} W^{\alpha}(x, \theta)+\frac{1}{2} N^{m n} \mathcal{F}_{m n}(x, \theta)
$$

satisfies $Q U=\partial\left(\lambda^{\alpha} A_{\alpha}\right)$ where $A_{m}=\frac{1}{8} D_{\alpha} \gamma_{m}^{\alpha \beta} A_{\beta}$ is the vector gauge superfield, $W^{\beta}=\frac{1}{10} \gamma_{m}^{\alpha \beta}\left(D_{\alpha} A^{m}-\right.$ $\left.\partial^{m} A_{\alpha}\right)$ is the spinor superfield strength, and $\mathcal{F}_{m n}=\frac{1}{8} D_{\alpha}\left(\gamma_{m n}\right)^{\alpha}{ }_{\beta} W^{\beta}=\partial_{[m} A_{n]}$ is the vector superfield strength. 


\section{Functional Integration, Picture-Changing Operators and the $b$ Ghost}

\subsection{Measure factor for pure spinor ghosts}

As reviewed in section (2.1), the gauge invariance of (2.4) implies that pure spinor ghosts can only appear through the operators $\lambda^{\alpha}, N_{m n}$ and $J$. Correlation functions for the non-zero modes of these operators are easily computed using the OPE's of (2.6). However, after integrating out the non-zero worldsheet modes, one still has to functionally integrate over the worldsheet zero modes. Because $\lambda^{\alpha}$ has zero conformal weight and satisfies the pure spinor constraint

$$
\lambda \gamma^{m} \lambda=0
$$

$\lambda^{\alpha}$ has 11 independent zero modes on a genus $g$ surface. And because $N_{m n}$ and $J$ have +1 conformal weight and are defined from gauge-invariant combinations of $w_{\alpha}$, they have $11 g$ independent zero modes on a genus $g$ surface. Note that (3.1) implies that $N_{m n}=\frac{1}{2}\left(w \gamma_{m n} \lambda\right)$ and $J=w \lambda$ are related by the equation [30]

$$
: N^{m n} \lambda^{\alpha}: \gamma_{m \alpha \beta}-\frac{1}{2}: J \lambda^{\alpha}: \gamma_{\alpha \beta}^{n}=2 \gamma_{\alpha \beta}^{n} \partial \lambda^{\alpha}
$$

where the normal-ordered product is defined by : $U^{A}(z) \lambda^{\alpha}(z):=\oint d y(y-z)^{-1} U^{A}(y) \lambda^{\alpha}(z)$. (The coefficient of the $\partial \lambda^{\alpha}$ term is determined by computing the double pole of the left-hand side of (3.2) with $J$.) Just as (3.1) implies that all 16 components of $\lambda^{\alpha}$ can be expressed in terms of 11 components, equation (3.2) implies that all 45 components of $N^{m n}$ can be expressed in terms of $J$ and ten components of $N^{m n}$.

Because of the constraints of (3.1) and (3.2), it is not immediately obvious how to functionally integrate over the pure spinor ghosts. However, as will be shown below, there is a natural Lorentz-invariant measure factor for the pure spinor ghosts which can be used to define functional integration.

A Lorentz-invariant measure factor for the $\lambda^{\alpha}$ zero modes can be obtained by noting that

$$
\left(d^{11} \lambda\right)^{\left[\alpha_{1} \alpha_{2} \ldots \alpha_{11}\right]} \equiv d \lambda^{\alpha_{1}} \wedge d \lambda^{\alpha_{2}} \wedge \ldots \wedge d \lambda^{\alpha_{11}}
$$

satisfies the identity

$$
\lambda^{\beta} \gamma_{\alpha_{1} \beta}^{m}\left(d^{11} \lambda\right)^{\left[\alpha_{1} \alpha_{2} \ldots \alpha_{11}\right]}=0
$$

because $\lambda \gamma^{m} d \lambda=0$. Using the properties of pure spinors, this implies that all $\frac{16 !}{5 ! 11 !}$ components of $\left(d^{11} \lambda\right)^{\left[\alpha_{1} \ldots \alpha_{11}\right]}$ are related to each other by a Lorentz-invariant measure factor $[\mathcal{D} \lambda]$ of +8 ghost number which is defined by

$$
\left(d^{11} \lambda\right)^{\left[\alpha_{1} \ldots \alpha_{11}\right]}=[\mathcal{D} \lambda] \mathcal{T}_{\left(\left(\beta_{1} \beta_{2} \beta_{3}\right)\right)}^{\left[\alpha_{1} \ldots \alpha_{11}\right]} \lambda^{\beta_{1}} \lambda^{\beta_{2}} \lambda^{\beta_{3}}
$$

where $\mathcal{T}_{\left(\left(\beta_{1} \beta_{2} \beta_{3}\right)\right)}^{\left[\alpha_{1} \ldots \alpha_{11}\right]}$ is the unique Lorentz-invariant tensor (up to rescaling) which is symmetric and $\gamma$-matrix traceless (i.e. $\gamma_{m}^{\beta_{1} \beta_{2}} \mathcal{T}_{\left(\left(\beta_{1} \beta_{2} \beta_{3}\right)\right)}^{\left[\alpha_{1} \ldots \alpha_{11}\right]}=0$ ) in three lowered indices and antisymmetric in eleven raised indices. It is defined by

$$
\begin{gathered}
\mathcal{T}_{\left(\left(\beta_{1} \beta_{2} \beta_{3}\right)\right)}^{\left[\alpha_{1} \alpha_{11}\right]} \\
=\varepsilon^{\alpha_{1} \ldots \alpha_{16}}\left(\gamma_{m n p}\right)_{\alpha_{12} \alpha_{13}}\left[\gamma_{\beta_{1} \alpha_{14}}^{m} \gamma_{\beta_{2} \alpha_{15}}^{n} \gamma_{\beta_{3} \alpha_{16}}^{p}-\frac{1}{40} \gamma_{q}^{\gamma \delta} \gamma_{\left(\beta_{1} \beta_{2}\right.}^{q} \gamma_{\left.\beta_{3}\right) \alpha_{14}}^{m} \gamma_{\gamma \alpha_{15}}^{n} \gamma_{\delta \alpha_{16}}^{p}\right] .
\end{gathered}
$$


One can similarly construct a Lorentz-invariant measure factor for the $N^{m n}$ and $J$ zero modes from

$$
\left(d^{11} N\right)^{\left[\left[m_{1} n_{1}\right]\left[m_{2} n_{2}\right] \ldots\left[m_{10} n_{10}\right]\right]} \equiv d N^{\left[m_{1} n_{1}\right]} \wedge d N^{\left[m_{2} n_{2}\right]} \wedge \ldots \wedge d N^{\left[m_{10} n_{10}\right]} \wedge d J
$$

Using the constraint of (3.2) and keeping $\lambda^{\alpha}$ fixed while varying $N^{m n}$ and $J$, one finds that (3.6) satisfies the identity

$$
\left(\lambda \gamma_{m_{1}}\right)_{\alpha}\left(d^{11} N\right)^{\left[\left[m_{1} n_{1}\right]\left[m_{2} n_{2}\right] \ldots\left[m_{10} n_{10}\right]\right]}=0 .
$$

Using the properties of pure spinors, this implies that all $\frac{45 !}{10 ! 35 !}$ components of

$$
\left(d^{11} N\right)^{\left[\left[m_{1} n_{1}\right]\left[m_{2} n_{2}\right] \ldots\left[m_{10} n_{10}\right]\right]}
$$

are related to each other by a Lorentz-invariant measure factor $[\mathcal{D N}]$ of -8 ghost number which is defined by

$$
\begin{gathered}
\left(d^{11} N\right)^{\left[\left[m_{1} n_{1}\right]\left[m_{2} n_{2}\right] \ldots\left[m_{10} n_{10}\right]\right]}=[\mathcal{D N}] \\
\left(\left(\lambda \gamma^{m_{1} n_{1} m_{2} m_{3} m_{4}} \lambda\right)\left(\lambda \gamma^{m_{5} n_{5} n_{2} m_{6} m_{7}} \lambda\right)\left(\lambda \gamma^{m_{8} n_{8} n_{3} n_{6} m_{9}} \lambda\right)\left(\lambda \gamma^{m_{10} n_{10} n_{4} n_{7} n_{9}} \lambda\right)\right. \\
+ \text { permutations })
\end{gathered}
$$

where the permutations are antisymmetric under the exchange of $m_{j}$ with $n_{j}$, and also antisymmetric under the exchange of $\left[m_{j} n_{j}\right]$ with $\left[m_{k} n_{k}\right]$. Note that the index structure on the right-hand side of (3.8) has been chosen such the expression is non-vanishing after summing over the permutations.

After using the OPE's of (2.6) to integrate out the non-zero modes of the pure spinor ghosts on a genus $g$ surface, one will obtain an expression

$$
\mathcal{A}=\left\langle f\left(\lambda, N_{1}, J_{1}, N_{2}, J_{2}, \ldots, N_{g}, J_{g}\right)\right\rangle
$$

which only depends on the 11 worldsheet zero modes of $\lambda$, and on the $11 g$ worldsheet zero modes of $N$ and $J$. Using the Lorentz-invariant measure factors defined in (3.5) and (3.8), the natural definition for functional integration over these zero modes is

$$
\mathcal{A}=\int[\mathcal{D} \lambda]\left[\mathcal{D} N_{1}\right]\left[\mathcal{D} N_{2}\right] \ldots\left[\mathcal{D} N_{g}\right] f\left(\lambda, N_{1}, J_{1}, N_{2}, J_{2}, \ldots, N_{g}, J_{g}\right)
$$

Note that with this definition, $f\left(\lambda, N_{1}, J_{1}, N_{2}, J_{2}, \ldots, N_{g}, J_{g}\right)$ must carry ghost number $-8+8 g$ to give a non-vanishing functional integral, which agrees with the -8 ghost-number anomaly in the OPE of $J$ with $T$. It will now be shown how the functional integral of (3.10) can be explicitly computed with the help of picture-changing operators.

\subsection{Picture-changing operators}

As is well-known from the work of Friedan-Martinec-Shenker [6] and Verlinde-Verlinde [2][3], picture-changing operators are necessary in the RNS formalism because of the bosonic $(\beta, \gamma)$ ghosts. Since the picture-raising and picture-lowering operators involve the delta functions $\delta(\beta)$ and $\delta(\gamma)$, insertion of these operators in loop amplitudes are needed to absorb the zero modes of the 
$(\beta, \gamma)$ ghosts on a genus $g$ surface. ${ }^{2}$ Up to possible surface terms, the amplitudes are independent of the worldsheet positions of these operators since the worldsheet derivatives of the picture-changing operators are BRST-trivial. The surface terms come from pulling the BRST operator through the $b$ ghosts to give total derivatives in the worldsheet moduli. If the correlation function diverges near the boundary of moduli space, these surface terms can give finite contributions which need to be treated carefully. As will now be shown, functional integration over the bosonic ghosts in the pure spinor formalism also requires picture-changing operators with similar properties to those of the RNS formalism. However, since the correlation functions in this formalism do not diverge near the boundary of moduli space, there are no subtleties due to surface terms.

To absorb the zero modes of $\lambda^{\alpha}, N_{m n}$ and $J$, picture-changing operators in the pure spinor formalism will involve the delta-functions $\delta\left(C_{\alpha} \lambda^{\alpha}\right), \delta\left(B_{m n} N^{m n}\right)$ and $\delta(J)$ where $C_{\alpha}$ and $B_{m n}$ are constant spinors and antisymmetric tensors. Although these constant spinors and tensors are needed for the construction of picture-changing operators, it will be shown that scattering amplitudes are independent of the choice of $C_{\alpha}$ and $B_{m n}$, so Lorentz invariance is preserved. As will be discussed later, this Lorentz invariance can be made manifest by integrating over all choices of $C_{\alpha}$ and $B_{m n}$. Note that the use of constant spinors and tensors in picture-changing operators is unrelated to the pure spinor constraint, and is necessary whenever the bosonic ghosts are not Lorentz scalars.

As in the RNS formalism, the picture-changing operators will be BRST-invariant with the property that their worldsheet derivative is BRST-trivial. A "picture-lowering" operator $Y_{C}$ with these properties is

$$
Y_{C}=C_{\alpha} \theta^{\alpha} \delta\left(C_{\beta} \lambda^{\beta}\right)
$$

where $C_{\alpha}$ is any constant spinor. Note that $Q Y_{C}=\left(C_{\alpha} \lambda^{\alpha}\right) \delta\left(C_{\beta} \lambda^{\beta}\right)=0$ and

$$
\partial Y_{C}=(C \partial \theta) \delta(C \lambda)+(C \theta)(C \partial \lambda) \delta^{\prime}(C \lambda)=Q\left[(C \partial \theta)(C \theta) \delta^{\prime}(C \lambda)\right]
$$

where $\delta^{\prime}(x) \equiv \frac{\partial}{\partial x} \delta(x)$ is defined using the usual rules for derivatives of delta functions, e.g. $x \delta^{\prime}(x)=$ $-\delta(x)$.

Although $Y_{C}$ is not spacetime-supersymmetric, its supersymmetry variation is BRST-trivial since

$$
q_{\alpha} Y_{C}=C_{\alpha} \delta(C \lambda)=-C_{\alpha}(C \lambda) \delta^{\prime}(C \lambda)=Q\left[-C_{\alpha}(C \theta) \delta^{\prime}(C \lambda)\right] .
$$

Similarly, $Y_{C}$ is not Lorentz invariant, but its Lorentz variation is BRST-trivial since

$$
\begin{aligned}
& M^{m n} Y_{C}= \frac{1}{2}\left(C \gamma^{m n} \theta\right) \delta(C \lambda)+\frac{1}{2}(C \theta)\left(C \gamma^{m n} \lambda\right) \delta^{\prime}(C \lambda) \\
&=Q\left[\frac{1}{2}\left(C \gamma^{m n} \theta\right)(C \theta) \delta^{\prime}(C \lambda)\right] .
\end{aligned}
$$

\footnotetext{
${ }^{2}$ In the RNS formalism, it is convenient to bosonize the $(\beta, \gamma)$ ghosts as $\beta=\partial \xi e^{-\phi}$ and $\gamma=\eta e^{\phi}$ since the spacetime supersymmetry generator involves a spin fi eld constructed for the negative-energy chiral boson $\phi$. The delta functions $\delta(\beta)$ and $\delta(\gamma)$ can then be expressed in terms of $\phi$ as $\delta(\beta)=e^{\phi}$ and $\delta(\gamma)=e^{-\phi}$. However, in the pure spinor formalism, there is no advantage to performing such a bosonization since all operators can be expressed directly in terms of $\lambda^{\alpha}$, $N^{m n}$ and $J$. Furthermore, since functional integration over the $\phi$ chiral boson can give rise to unphysical poles in the correlation functions, the fact that all operators in the pure spinor formalism can be expressed in terms of $\left(\lambda^{\alpha}, N^{m n}, J\right)$ allows one to avoid unphysical poles in pure spinor correlation functions.
} 
So different choices of $C_{\alpha}$ only change $Y_{C}$ by a BRST-trivial quantity, and any on-shell amplitude computations involving insertions of $Y_{C}$ will be Lorentz invariant and spacetime supersymmetric up to possible surface terms. The fact that Lorentz invariance is preserved only up to surface terms is unrelated to the pure spinor constraint, and is caused by the bosonic ghosts not being Lorentz scalars.

One can also construct BRST-invariant operators involving $\delta\left(B^{m n} N_{m n}\right)$ and $\delta(J)$ with the property that their worldsheet derivative is BRST-trivial. These "picture-raising" operators will be called $Z_{B}$ and $Z_{J}$ and are defined by

$$
Z_{B}=\frac{1}{2} B_{m n}\left(\lambda \gamma^{m n} d\right) \delta\left(B^{p q} N_{p q}\right), \quad Z_{J}=\left(\lambda^{\alpha} d_{\alpha}\right) \delta(J),
$$

where $B_{m n}$ is a constant antisymmetric tensor. One can check that $Q Z_{B}=Q Z_{J}=0$ and that $\partial Z_{B}$ and $\partial Z_{J}$ are BRST-trivial. Furthermore, different choices of $B_{m n}$ only change $Z_{B}$ by a BRST-trivial quantity.

\subsection{Construction of $b$ Ghost}

To compute $g$-loop amplitudes, the usual string theory prescription requires the insertion of $(3 g-3) b$ ghosts of -1 ghost-number which satisfy

$$
\{Q, b(u)\}=T(u)
$$

where $T$ is the stress tensor of (2.7). After integrating $b(u)$ with a Beltrami differential $\mu_{P}(u)$ for $P=1$ to $3 g-3$, the BRST variation of $b(u)$ generates a total derivative with respect to the Teichmuller parameter $\tau_{P}$ associated to the Beltrami differential $\mu_{P}$. But since $w_{\alpha}$ can only appear in gauge-invariant combinations of zero ghost number, there are no operators of negative ghost number in the pure spinor formalism, so one cannot construct such a $b$ ghost. Nevertheless, as will now be shown, the picture-raising operator

$$
Z_{B}=\frac{1}{2} B_{m n}\left(\lambda \gamma^{m n} d\right) \delta(B N)
$$

can be used to construct a suitable substitute for the $b$ ghost in non-zero picture.

Since genus $g$ amplitudes also require $10 g$ insertions of $Z_{B}(z)$, one can combine $(3 g-3)$ insertions of $Z_{B}(z)$ with the desired insertions of the $b(u)$ ghost and look for a non-local operator $\widetilde{b}_{B}(u, z)$ which satisfies

$$
\left\{Q, \widetilde{b}_{B}(u, z)\right\}=T(u) Z_{B}(z) .
$$

Note that $Z_{B}$ carries +1 ghost-number, so $\widetilde{b}_{B}$ carries zero ghost number. And (3.17) implies that integrating $\widetilde{b}(u, z)$ with the Beltrami differential $\mu_{P}(u)$ has the same properties as integrating $b(u)$ with $\mu_{P}(u)$ in the presence of a picture-raising operator $Z_{B}(z)$.

Using

$$
Z_{B}(z)=Z_{B}(u)+\int_{u}^{z} d v \partial Z_{B}(v)=Z_{B}(u)+\int_{u}^{z} d v\left\{Q, B_{p q} \partial N^{p q}(v) \delta(B N(v))\right\},
$$

one can define

$$
\widetilde{b}_{B}(u, z)=b_{B}(u)+T(u) \int_{u}^{z} d v B_{p q} \partial N^{p q}(v) \delta(B N(v))
$$


where $b_{B}(u)$ is a local operator satisfying

$$
\left\{Q, b_{B}(u)\right\}=T(u) Z_{B}(u) .
$$

The explicit formula for $b_{B}(u)$ satisfying (3.19) is complicated and was computed in ([22]) up to some undetermined coefficients. Ignoring Lorentz indices, $b_{B}$ has the form

$$
\begin{gathered}
b_{B}=B\left(d^{2} \Pi+d N \partial \theta+N^{2}+N \Pi^{2}\right) \delta(B N) \\
+B^{2}\left(d^{4}+d^{2} N \Pi+N^{2} \Pi^{2}+N^{2} d \partial \theta\right) \delta^{\prime}(B N) \\
+B^{3}\left(d^{4} N+d^{2} N^{2} \Pi\right) \delta^{\prime \prime}(B N)+B^{4}\left(d^{4} N^{2}\right) \delta^{\prime \prime \prime}(B N) .
\end{gathered}
$$

For proving vanishing theorems, it will be useful to note that all terms in $b_{B}$ have +2 conformal weight and +4 "engineering" dimension where $[\lambda, \theta, x, d, N]$ are defined to carry $\left[0, \frac{1}{2}, 1, \frac{3}{2}, 2\right]$ engineering dimension and $\delta(B N)$ carries -1 conformal weight and zero engineering dimension.

\section{Multiloop Amplitude Prescription}

Using the picture-changing operators and $b_{B}$ ghost of section 3 , one can define a superPoincaré covariant prescription for computing $N$-point $g$-loop closed superstring scattering amplitudes as

$$
\begin{gathered}
\mathcal{A}=\int d^{2} \tau_{1} \ldots d^{2} \tau_{3 g-3}\langle| \prod_{P=1}^{3 g-3} \int d^{2} u_{P} \mu_{P}\left(u_{P}\right) \widetilde{b}_{B_{P}}\left(u_{P}, z_{P}\right) \\
\left.\left.\prod_{P=3 g-2}^{10 g} Z_{B_{P}}\left(z_{P}\right) \prod_{R=1}^{g} Z_{J}\left(v_{R}\right) \prod_{I=1}^{11} Y_{C_{I}}\left(y_{I}\right)\right|^{2} \prod_{T=1}^{N} \int d^{2} t_{T} U_{T}\left(t_{T}\right)\right\rangle,
\end{gathered}
$$

where ||$^{2}$ signifies the left-right product, $\tau_{P}$ are the Teichmuller parameters associated to the Beltrami differentials $\mu_{P}\left(u_{P}\right)$, and $U_{T}\left(t_{T}\right)$ are the dimension $(1,1)$ closed string vertex operators for the $N$ external states. The number of picture-lowering and picture-raising operators in (4.1) are appropriate for absorbing the 11 zero modes of $\lambda^{\alpha}$ and the $11 \mathrm{~g}$ zero modes of $w_{\alpha}$, and the locations of these picture-changing operators can be chosen arbitrarily. The constant antisymmetric tensors $B_{P}^{m n}$ in $b_{B_{P}}$ and $Z_{B_{P}}$ will be chosen such that $B_{I}=B_{I+10}=\ldots=B_{I+10(g-1)}$ for $I=1$ to 10 . In other words, there will be ten constant antisymmetric tensors $B_{I}^{m n}$, each of which appear in $g$ pictureraising operators or $b_{B}$ ghosts.

When $g=1$, the prescription of (4.1) needs to be modified for the usual reason that genusone worldsheets are invariant under constant translations, so one of the vertex operators should be unintegrated. The one-loop amplitude prescription is therefore

$$
\begin{gathered}
\mathcal{A}=\int d^{2} \tau\langle| \int d^{2} u \mu(u) \widetilde{b}_{B_{1}}\left(u, z_{1}\right) \\
\left.\left.\prod_{P=2}^{10} Z_{B_{P}}\left(z_{P}\right) Z_{J}(v) \prod_{I=1}^{11} Y_{C_{I}}\left(y_{I}\right)\right|^{2} V_{1}\left(t_{1}\right) \prod_{T=2}^{N} \int d^{2} t_{T} U_{T}\left(t_{T}\right)\right\rangle,
\end{gathered}
$$


where $V_{1}\left(t_{1}\right)$ is the unintegrated closed string vertex operator. And when $g=0$, three of the vertex operators are unintegrated and one uses the prescription

$$
\mathcal{A}=\left\langle\left|\prod_{I=1}^{11} Y_{C_{I}}\left(y_{I}\right)\right|^{2} V_{1}\left(t_{1}\right) V_{2}\left(t_{2}\right) V_{3}\left(t_{3}\right) \prod_{T=4}^{N} \int d^{2} t_{T} U_{T}\left(t_{T}\right)\right\rangle .
$$

As discussed in section 3, the Lorentz variations of $\widetilde{b}_{B_{P}}, Z_{B_{P}}$ and $Y_{C_{I}}$ are BRST-trivial, so the prescription is Lorentz-invariant up to possible surface terms. Also, all operators are manifestly spacetime supersymmetric except for $Y_{C_{I}}$, whose supersymmetry variation is BRST-trivial. In section 5, it will be argued that surface terms can be ignored in this formalism because of finiteness properties of the correlation functions. So the amplitude prescriptions of (4.1), (4.2) and (4.3) are super-Poincare covariant and $\mathcal{A}$ is independent of the eleven constant spinors $C_{I}$ and ten constant tensors $B_{P}$ which appear in the picture-changing operators. One can therefore obtain manifestly Lorentz-covariant expressions from this amplitude prescription by functionally integrating over the matter fields and pure spinor ghosts.

As usual, the functional integration factorizes into partition functions and correlation functions for the different worldsheet variables. However, in the pure spinor formalism, the partition functions for the different worldsheet variables cancel each other out. This is easy to verify since the partition function for the ten bosonic $x^{\mu}$ variables gives a factor of $\left(\operatorname{det} \bar{\partial}_{0}\right)^{-5}\left(\operatorname{det} \partial_{0}\right)^{-5}$ where $\partial_{0}$ and $\bar{\partial}_{0}$ are the holomorphic and antiholomorphic derivatives acting on fields of zero conformal weight, the partition function for the sixteen fermionic $\left(\theta^{\alpha}, p_{\alpha}\right)$ and $\left(\bar{\theta}^{\alpha}, \bar{p}_{\alpha}\right)$ variables gives a factor of $\left(\operatorname{det} \bar{\partial}_{0}\right)^{16}\left(\operatorname{det} \partial_{0}\right)^{16}$, and the partition function for the eleven bosonic $\left(\lambda^{\alpha}, w_{\alpha}\right)$ and $\left(\bar{\lambda}^{\alpha}, \bar{w}_{\alpha}\right)$ variables gives a factor of $\left(\operatorname{det} \bar{\partial}_{0}\right)^{-11}\left(\operatorname{det} \partial_{0}\right)^{-11}$. So to perform the functional integral, one only needs to compute the correlation functions for the matter variables and pure spinor ghosts.

As described in detail in [22], these correlation functions can be computed by first separating off the zero modes from the worldsheet variables and then using the OPE's of (2.6) and (2.10) for performing the correlation functions for the nonzero modes of these variables. Finally, one integrates over the worldsheet zero modes using the usual measure factors for the matter variables $\left(x^{m}, \theta^{\alpha}, p_{\alpha}\right)$ and using the Lorentz-invariant measure factors of subsection (3.1) for the pure spinor ghost variables.

The resulting expression for the scattering amplitude naively depends on the eleven constant spinors $C_{I}$ and ten constant tensors $B_{P}$ which appear in the picture-changing operators. However, due to Lorentz invariance of the picture-changing operators, one is guaranteed that this dependence on $C_{I}$ and $B_{P}$ is BRST-trivial. One can therefore integrate over all possible choices of $C_{I}$ and $B_{P}$ and obtain a manifestly Lorentz-covariant expression for the multiloop amplitude. As shown in [22], integration over $C_{I}$ and $B_{P}$ is straightforward and the resulting covariant expression agrees for tree amplitudes and for massless four-point one-loop amplitudes with the well-known RNS expression.

\section{Vanishing Theorems}

In this section, the amplitude prescription of section 4 will be used to prove certain vanishing theorems for massless closed superstring scattering amplitudes. In subsection (5.1), it will be proven that massless $N$-point $g$-loop amplitudes are vanishing whenever $N<4$ and $g>0$, implying (with two mild assumptions) the perturbative finiteness of superstring theory. And in subsection 
(5.2), it will be proven that the low-energy limit of the four-point massless amplitude gets no perturbative contributions above one-loop, in agreement with the Type IIB S-duality conjecture of Green and Gutperle.

To prove these vanishing theorems, it will be useful to express the massless closed superstring vertex operator as the left-right product of two open superstring vertex operators as $V_{\text {closed }}=V_{\text {open }} \times$ $\bar{V}_{\text {open }}$ where the closed superstring graviton $h^{m n}$, gravitini $\psi_{m}^{\alpha}$ and $\bar{\psi}_{m}^{\alpha}$, and Ramond-Ramond field strength $F^{\alpha \beta}$ are identified with left-right products of the open superstring photon $a_{m}$ and photino $\xi^{\alpha}$ as

$$
h_{m n}=a_{m} \bar{a}_{n}, \quad \psi_{m}^{\alpha}=a_{m} \bar{\xi}^{\alpha}, \quad \bar{\Psi}_{m}^{\alpha}=\xi^{\alpha} \bar{a}_{m}, \quad F^{\alpha \beta}=\xi^{\alpha \bar{\xi}^{\beta}} .
$$

Using the unintegrated and integrated open superstring massless vertex operators of (2.12) and (2.16), this implies that

$$
\begin{gathered}
V_{\text {closed }}=\lambda^{\alpha} \bar{\lambda}^{\beta} A_{\alpha \beta}(x, \theta, \bar{\theta})=e^{i k \cdot x} \lambda^{\alpha} A_{\alpha}(\theta) \bar{\lambda}^{\beta} \bar{A}_{\beta}(\bar{\theta}) \quad \text { and } \\
U_{\text {closed }}=e^{i k \cdot x}\left(\partial \theta^{\alpha} A_{\alpha}(\theta)+\Pi^{m} A_{m}(\theta)+d_{\alpha} W^{\alpha}(\theta)+\frac{1}{2} N^{m n} \mathcal{F}_{m n}(\theta)\right) \\
\left(\bar{\partial} \bar{\theta}^{\beta} \bar{A}_{\beta}(\bar{\theta})+\bar{\Pi}^{p} \bar{A}_{p}(\bar{\theta})+\bar{d}_{\beta} \bar{W}^{\beta}(\bar{\theta})+\frac{1}{2} \bar{N}^{p q} \overline{\mathcal{F}}_{p q}(\bar{\theta})\right)
\end{gathered}
$$

are the unintegrated and integrated massless closed superstring vertex operators.

\subsection{Non-renormalization theorem}

In this subsection, the amplitude prescription of section 4 will be used to prove that massless $N$-point $g$-loop amplitudes vanish whenever $N<4$ and $g>0$. For $N=0$, this implies vanishing of the cosmological constant; for $N=1$, it implies absence of tadpoles; for $N=2$, it implies the mass is not renormalized; and for $N=3$, it implies the coupling constant is not renormalized. Using the arguments of [4][24] which were summarized in the introduction, and assuming factorization and the absence of unphysical divergences in the interior of moduli space, these non-renormalization theorems imply that massless superstring scattering amplitudes are finite order-by-order in perturbation theory.

Although surface terms were ignored in deriving the amplitude prescription of section 4 , it is necessary that the proof of the non-renormalization theorem remain valid even if one includes such surface term contributions. Otherwise, there could be divergent surface term contributions which would invalidate the proof. For this reason, one cannot assume Lorentz invariance or spacetime supersymmetry to prove the non-renormalization theorem since the prescription of (4.1) is Lorentz invariant and spacetime supersymmetric only after ignoring the surface terms.

Fortunately, it will be possible to prove the non-renormalization theorem using only the counting of zero modes. Since this type of argument implies the pointwise vanishing of the integrand of the scattering amplitude (as opposed to only implying that the integrated amplitude vanishes), the proof remains valid if one includes the contribution of surface terms.

On a surface of arbitrary genus, one needs 16 zero modes of $\theta^{\alpha}$ and $\bar{\theta}^{\alpha}$ for the amplitude to be non-vanishing. Since the only operators in (4.1) containing $\theta^{\alpha}$ zero modes ${ }^{3}$ are the eleven $Y_{C}$

\footnotetext{
${ }^{3}$ When expressed in terms of the free fi elds $\left(x^{m}, \theta^{\alpha}, p_{\alpha}\right), \Pi^{m}$ and $d_{\alpha}$ contain $\theta$ 's without derivatives which naively could contribute $\theta^{\alpha}$ zero modes. But if the supersymmetric OPE's of (2.10) are used to integrate out the non-zero worldsheet modes, the OPE's involving $\Pi^{m}$ and $d_{\alpha}$ will never produce $\theta^{\alpha}$ zero modes.
} 
picture-lowering operators and the $U_{T}$ vertex operators, and since each $Y_{C}$ contributes a single $\theta^{\alpha}$ zero mode, the $U_{T}$ vertex operators must contribute at least five $\theta^{\alpha}$ and five $\bar{\theta}^{\alpha}$ zero modes for the amplitude to be non-vanishing. This immediately implies that zero-point amplitudes vanish.

For one-point amplitudes, conservation of momentum implies that the external state must have momentum $k^{m}=0$. But when $k^{m}=0$, the maximum number of zero modes in the vertex operator is one $\theta^{\alpha}$ and one $\bar{\theta}^{\alpha}$ coming from the superfield

$$
A_{\alpha \beta}(\theta, \bar{\theta})=h_{m n}\left(\gamma^{m} \theta\right)_{\alpha}\left(\gamma^{n} \bar{\theta}\right)_{\beta} .
$$

All other components in the superfields appearing in the vertex operators of (5.1) and (5.2) are either fermionic or involve powers of $k^{m}$. So all one-point amplitudes vanish.

To prove that massless two and three-point amplitudes vanish for non-zero $g$, one needs to count the available zero modes of $d_{\alpha}$, as well as the zero modes of $N_{m n}$. On a genus $g$ surface, nonvanishing amplitudes require $16 \mathrm{~g}$ zero modes of $d_{\alpha}$. In addition, the number of $N_{m n}$ zero modes must be at least as large as the number of derivatives acting on the delta functions $\delta(B N)$ in the amplitude prescription. Otherwise, integration over the $N^{m n}$ zero modes will trivially vanish.

To prove the $N$-point $g$-loop non-renormalization theorem for $N=2$ and $N=3$, it is useful to distinguish between one-loop amplitudes and multiloop amplitudes. For massless $N$-point oneloop amplitudes using the prescription of (4.2), there are $(N-1)$ integrated vertex operators of (5.2), each of which can either provide a $d_{\alpha}$ zero mode or an $N_{m n}$ zero mode. So one has at most $(N-1-M) d_{\alpha}$ zero modes and $M N_{m n}$ zero modes coming from the vertex operators where $M \leq N-1$. Each of the nine $Z_{B_{P}}$ operators and one $Z_{J}$ operator can provide a single $d_{\alpha}$ zero mode, so to get a total of $16 d_{\alpha}$ zero modes, $b_{B}$ must provide at least

$$
16-(N-1-M)-9-1=7-N+M
$$

$d_{\alpha}$ zero modes.

It is easy to verify from (3.20) that $b_{B}$ can provide a maximum of four $d_{\alpha}$ zero modes, however, the terms containing four $d_{\alpha}$ zero modes also contain $(-1) N_{m n}$ zero modes where a derivative acting on $\delta(B N)$ counts as a negative $N_{m n}$ zero mode. This fact can easily be derived from the +4 engineering dimension of $b_{B}$ where $\left[\lambda^{\alpha}, \theta^{\alpha}, x^{m}, d_{\alpha}, N_{m n}\right]$ are defined to carry engineering dimension $\left[0, \frac{1}{2}, 1, \frac{3}{2}, 2\right]$ and $\delta(B N)$ is defined to carry zero engineering dimension. Since $(d)^{4}$ carries engineering dimension +6 , it can only appear in $b_{B}$ together with a term such as $\delta^{\prime}(B N)$ which carries engineering dimension -2 .

So for $N \leq 3$ and $M=0$, (5.3) implies that the only way to obtain $16 d_{\alpha}$ zero modes is if $b_{B}$ provides at least four $d_{\alpha}$ zero modes. But in this case, $b_{B}$ contains $(-1) N_{m n}$ zero modes, so the amplitudes vanish since there are not enough $N_{m n}$ zero modes to absorb the derivatives on $\delta(B N)$. And when $M>0$, the amplitude vanishes for $N \leq 3$ since one needs more than four $d_{\alpha}$ zero modes to come from $b_{B}$.

For multiloop amplitudes, the argument is similar, but one now has $N$ integrated vertex operators instead of $(N-1)$. So the vertex operators can contribute a maximum of $(N-M) d_{\alpha}$ zero modes and $M N_{m n}$ zero modes where $M \leq N$. And each of the $7 g+3 Z_{B}$ and $g Z_{J}$ operators can provide a single $d_{\alpha}$ zero mode. So to get a total of $16 g d_{\alpha}$ zero modes, the $(3 g-3) b_{B}$ 's must provide at least

$$
16 g-(N-M)-(7 g+3)-g=8 g-3-N+M
$$


$d_{\alpha}$ zero modes. Since $(3 g-3) b_{B}$ 's carry engineering dimension $12 g-12, d_{\alpha}$ carries engineering dimension $\frac{3}{2}$, and $N_{m n}$ carries engineering dimension +2 , the $(3 g-3) b_{B}$ 's can provide a maximum of $(8 g-8) d_{\alpha}$ zero modes with no derivatives of $\delta(B N)$, or $\left(8 g-8+\frac{4}{3} M\right) d_{\alpha}$ zero modes with $M$ derivatives of $\delta(B N)$. Since

$$
8 g-8+\frac{4}{3} M<8 g-3-N+M
$$

whenever $M \leq N \leq 3$, there is no way for the $(3 g-3) b_{B}$ 's to provide enough $d_{\alpha}$ zero modes without providing too many derivatives of $\delta(B N)$.

So the $N$-point multiloop non-renormalization theorem has been proven for $N \leq 3$. Note that when $N=4$,

$$
8 g-8+\frac{4}{3} M \geq 8 g-3-N+M
$$

if one chooses $M=3$ or $M=4$. So four-point multiloop amplitudes do not need to vanish. However, as will be shown in subsection (5.2), one can prove that the low-energy limit of these multiloop amplitudes vanish, which implies that the $R^{4}$ term in the effective action gets no perturbative corrections above one loop.

\subsection{Absence of multiloop $R^{4}$ contributions}

Although the four-point massless amplitude is expected to be non-vanishing at all loops, there is a conjecture based on S-duality of the Type IIB effective action that $R^{4}$ terms in the low-energy effective action do not get perturbative contributions above one-loop [23]. After much effort, this conjecture was recently verified in the RNS formalism at two loops [7][5]. As will now be shown, the multiloop prescription of section 4 can be easily used to prove the validity of this S-duality conjecture at all loops.

It was proven using (5.6) that the four-point massless multiloop amplitude vanishes unless at least three of the four integrated vertex operators contribute an $N_{m n}$ zero mode. Since the only operators containing $\theta$ zero modes are the eleven picture-lowering operators and the external vertex operators, the functional integral over $\theta$ zero modes in the multiloop prescription for the four-point amplitude gives an expression of the form

$$
\left|\int d^{16} \theta(\theta)^{11}\left(d_{\alpha} W_{1}^{\alpha}(\theta)+\frac{1}{2} N_{p q} \mathcal{F}_{1}^{p q}(\theta)\right) \prod_{T=2}^{4} N_{m n} \mathcal{F}_{T}^{m n}(\theta)\right|^{2} .
$$

Since the external vertex operators must contribute at least $5 \theta^{\alpha}$ and $\bar{\theta}^{\alpha}$ zero modes, one easily sees that there is no way to produce an $\left|F^{4}\right|^{2}$ term which would imply an $R^{4}$ term in the effective action. In fact, by examining the component expansion of the $\mathcal{F}_{m n}(\theta)$ and $W^{\alpha}(\theta)$ superfields, one finds that the term with fewest number of spacetime derivatives which contributes $5 \theta$ 's and $5 \bar{\theta}$ 's is $\left|(\partial F)(\partial F) F^{2}\right|^{2}$, which would imply a $\partial^{4} R^{4}$ contribution to the low-energy effective action.

So it has been proven that there are no multiloop contributions to $R^{4}$ terms (or $\partial^{2} R^{4}$ terms) in the low-energy effective action of the superstring. It should be noted that this proof has assumed that the correlation function over $x^{m}$ does not contribute inverse powers of $k^{m}$ which could cancel momentum factors coming from the $\theta$ integration in (5.7). Although the $x^{m}$ correlation function does contain poles as a function of $k^{m}$ when the external vertex operators collide, these poles only contribute to non-local terms in the effective action which involve massless propagators, and are not expected to contribute to local terms in the effective action such as the $R^{4}$ term. 


\section{Conclusions}

As discussed in these proceedings, the super-Poincaré covariant prescription for multiloop superstring amplitudes has several advantages over the RNS prescription. There is no sum over spin structures, surface terms from the boundary of moduli space can be ignored, and there are no unphysical poles from a negative-energy chiral boson. Furthermore, the partition functions for the matter and ghost variables cancel, amplitudes involving external Ramond states are no more complicated than those involving external Neveu-Schwarz states, and one can easily prove certain vanishing theorems by counting zero modes of the fermionic superspace variables.

Nevertheless, there are still some open questions concerning the super-Poincaré covariant prescription which would be useful to answer. Since the formalism has only been defined in conformal gauge, it is not yet clear how to derive the BRST operator and picture-changing operators from a worldsheet reparameterization-invariant action analogous to the Nambu-Goto action for the bosonic string. One clue may come from the $\mathrm{N}=2$ twistor-string formalism which has been shown at the classical level to be related to the pure spinor formalism and the $b$ ghost [31]. Another important question is to show that the multiloop prescription is unitary, possibly by proving its equivalence with a light-cone gauge prescription.

There are many possible applications of the multiloop prescription described here. For example, one could try to verify duality conjectures which imply vanishing theorems for higherderivative $R^{4}$ terms [32], $R^{4} H^{4 g-4}$ terms [33], and $F^{2 n}$ terms [34]. Another possible application is to generalize multiloop computations in a flat ten-dimensional background to multiloop computations in a Calabi-Yau background, perhaps by using the hybrid formalism. Finally, a recent exciting application of these methods has been developed by Anguelova, Grassi and Vanhove [35] for computing covariant one-loop amplitudes in eleven dimensions using the pure spinor version of the $d=11$ superparticle [36].

Acknowledgements: I would like to thank the organizers of the Fourth International Winter Conference for the invitation to speak, Sergey Cherkis, Mike Douglas, Michael Green, Warren Siegel, Mario Tonin, Brenno Carlini Vallilo, Pierre Vanhove, Herman Verlinde and Edward Witten for useful discussions, CNPq grant 300256/94-9, Pronex 66.2002/1998-9, and FAPESP grant 99/12763-0 for partial financial support, and the Institute of Advanced Studies and Fundação Instituto de Física Teórica for their hospitality.

\section{References}

[1] O. Lechtenfeld and A. Parkes, On Covariant Multiloop Superstring Amplitudes, Nucl. Phys. B332 (1990) 39 .

[2] E. Verlinde and H. Verlinde, Multiloop Calculations in Covariant Superstring Theory, Phys. Lett. B192 (1987) 95.

[3] H. Verlinde, The Path Integral Formulation of Supersymmetric String Theory, PhD Thesis, Univ. of Utrecht (1988).

[4] J. Atick, G. Moore, and A. Sen, Catoptric Tadpoles, Nucl. Phys. B307 (1988) 221. 
[5] E. D’Hoker and D.H. Phong, Two Loop Superstrings, 1. Main Formulas, Phys. Lett. B529 (2002) 241, hep-th/0110247.

[6] D. Friedan, E. Martinec and S. Shenker, Conformal Invariance, Supersymmetry and String Theory, Nucl. Phys. B271 (1986) 93.

[7] R. Iengo and C.-J. Zhu, Two Loop Computation of the Four-Particle Amplitude in the Heterotic String, Phys. Lett. B212 (1988) 313; R. Iengo and C.-J. Zhu, Explicit Modular Invariant Two-Loop Superstring Amplitude Relevant for $R^{4}$, JHEP 06 (1999) 011, hep-th/9905050.

[8] M.B. Green and J.H. Schwarz, Superstring Interactions, Nucl. Phys. B218 (1983) 43; M.B. Green and J.H. Schwarz, Superstring Field Theory, Nucl. Phys. B243 (1984) 475.

[9] S. Mandelstam, Interacting String Picture of the Neveu-Schwarz-Ramond Model, Nucl. Phys. B69 (1974) 77.

[10] S. Mandelstam, Interacting String Picture of the Fermionic String, Prog. Theor. Phys. Suppl. 86 (1986) 163.

[11] J. Greensite and F.R. Klinkhamer, Superstring Amplitudes and Contact Interactions, Nucl. Phys. B304 (1988) 108; M.B. Green and N. Seiberg, Contact Interactions in Superstring Theory, Nucl. Phys. B299 (1988) 559.

[12] N. Berkovits, Super-Poincaré Covariant Quantization of the Superstring, JHEP 04 (2000) 018, hep-th/0001035.

[13] N. Berkovits, ICTP Lectures on Covariant Quantization of the Superstring, hep-th/0209059.

[14] N. Berkovits and B.C. Vallilo, Consistency of Super-Poincaré Covariant Superstring Tree Amplitudes, JHEP 07 (2000) 015, hep-th/0004171.

[15] N. Berkovits, Relating the RNS and Pure Spinor Formalisms for the Superstring, JHEP 08 (2001) 026, hep-th/0104247..

[16] N. Berkovits, Cohomology in the Pure Spinor Formalism for the Superstring, JHEP 09 (2000) 046, hep-th/0006003; N. Berkovits and O. Chandía, Lorentz Invariance of the Pure Spinor BRST Cohomology for the Superstring, Phys. Lett. B514 (2001) 394, hep-th/0105149.

[17] N. Berkovits and P. Howe, Ten-Dimensional Supergravity Constraints from the Pure Spinor Formalism for the Superstring Nucl. Phys. B635 (2002) 75, hep-th/0112160.

[18] B.C. Vallilo and O. Chandía, Conformal Invariance of the Pure Spinor Superstring in a Curved Background, JHEP 0404 (2004) 041, hep-th/0401226.

[19] P.A. Grassi, G. Policastro, M. Porrati and P. Van Nieuwenhuizen, Covariant Quantization of Superstrings without Pure Spinor Constraints, JHEP 0210 (2002) 054, hep-th/0112162; P.A. Grassi, G. Policastro and P. Van Nieuwenhuizen, An Introduction to the Covariant Quantization of Superstrings, Class. Quant. Grav. 20 (2003) S395, hep-th/0302147.

[20] Y. Aisaka and Y. Kazama, A New First Class Algebra, Homological Perturbation and Extension of Pure Spinor Formalism for Superstring, JHEP 0302 (2003) 017, hep-th/0212316; Y. Aisaka and Y. Kazama, Operator Mapping between RNS and Extended Pure Spinor Formalisms for Superstring, JHEP 0308 (2003) 047, hep-th/0305221.

[21] M. Chesterman, Ghost Constraints and the Covariant Quantization of the Superparticle in Ten Dimensions, JHEP 0402 (2004) 011, hep-th/0212261; M. Chesterman, On the Cohomology and Inner Products of the Berkovits Superparticle and Superstring, hep-th/0404021. 
[22] N. Berkovits, Multiloop Amplitudes and Vanishing Theorems using the Pure Spinor Formalism for the Superstring, to appear in JHEP, hep-th/0406055.

[23] M.B. Green and M. Gutperle, Effects of D Instantons, Nucl. Phys. B498 (1997) 195, hep-th/9701093; M.B. Green and P. Vanhove, D-instantons, Strings and M-theory, Phys. Lett. B408 (1997) 122, hep-th/9704145.

[24] E. Martinec, Nonrenormalization Theorems and Fermionic String Finiteness, Phys. Lett. B171 (1986) 189.

[25] S. Mandelstam, The n Loop String Amplitude: Explicit Formulas, Finiteness and Absence of Ambiguities, Phys. Lett. B277 (1992) 82.

[26] A. Restuccia and J.G. Taylor, Finiteness of Type II Superstring Amplitudes, Phys. Lett. B187 (1987) 267; R. Kallosh and A. Morozov, On Vanishing of Multiloop Contributions to 0,1,2,3 Point Functions in Green-Schwarz Formalism for Heterotic String, Phys. Lett. B207 (1988) 164.

[27] M.B. Green and J.H. Schwarz, Covariant Description of Superstrings, Phys. Lett. B131 (1984) 367.

[28] W. Siegel, Classical Superstring Mechanics, Nucl. Phys. B263 (1986) 93.

[29] P. Howe, Pure Spinor Lines in Superspace and Ten-Dimensional Supersymmetric Theories, Phys. Lett. B258 (1991) 141.

[30] N. Berkovits and O. Chandía, Massive Superstring Vertex Operator in D=10 Superspace, JHEP 0208 (2002) 040, hep-th/0204121.

[31] M. Matone, L. Mazzucato, I. Oda, D. Sorokin and M. Tonin, The Superembedding Origin of the Berkovits Pure Spinor Covariant Quantization of Superstrings, Nucl. Phys. B639 (2002) 182, hep-th/0206104; I. Oda and M. Tonin, On the B Antighost in the Pure Spinor Quantization of Superstrings, hep-th/0409052.

[32] M.B. Green, H-h. Kwon and P. Vanhove, Two Loops in Eleven Dimensions, Phys. Rev. D61 (2000) 104010, hep-th/9910055.

[33] N. Berkovits and C. Vafa, Type IIB $R^{4} H^{4 g-4}$ Conjectures, Nucl. Phys. B533 (1998) 181, hep-th/9803145.

[34] S. Stieberger and T.R. Taylor, Nonabelian Born-Infeld Action and Type I - Heterotic Duality 2: Nonrenormalization Theorems, Nucl. Phys. B648 (2003) 3, hep-th/0209064.

[35] L. Anguelova, P.A. Grassi and P. Vanhove, Covariant One-Loop Amplitudes in D=11, hep-th/0408171.

[36] N. Berkovits, Towards Covariant Quantization of the Supermembrane, JHEP 0209 (2002) 051, hep-th/0201151. 